Proc. Indian Acad. Sci. (Math. Sci.) Vol. 115, No. 3, August 2003, pp. 251-257.

Printed in India

\title{
Homeomorphisms and the homology of non-orientable surfaces
}

\author{
SIDDHARTHA GADGIL and DISHANT PANCHOLI ${ }^{*}$
}

Stat Math Unit, Indian Statistical Institute, Bangalore 560 059, India

${ }^{*}$ School of Mathematics, Tata Institute of Fundamental Research, Mumbai 400 005,

India

E-mail: gadgil@isibang.ac.in; dishant@math.tifr.res.in

MS received 8 February 2005; revised 3 May 2005

\begin{abstract}
We show that, for a closed non-orientable surface $F$, an automorphism of $H_{1}(F, \mathbb{Z})$ is induced by a homeomorphism of $F$ if and only if it preserves the $(\bmod 2)$ intersection pairing. We shall also prove the corresponding result for punctured surfaces.
\end{abstract}

Keywords. Non-orientable surfaces; Dehn twist; mapping class groups; crosscap slide.

\section{Introduction}

Let $F$ be a closed, non-orientable surface. A homeomorphism $f: F \rightarrow F$ induces an automorphism on homology $f_{*}: H_{1}(F, \mathbb{Z}) \rightarrow H_{1}(F, \mathbb{Z})$. Further, any automorphism $\varphi: H_{1}(F, \mathbb{Z}) \rightarrow H_{1}(F, \mathbb{Z})$ in turn induces an automorphism with $\mathbb{Z} / 2 \mathbb{Z}$-coefficients $\bar{\varphi}: H_{1}(F, \mathbb{Z} / 2 \mathbb{Z}) \rightarrow H_{1}(F, \mathbb{Z} / 2 \mathbb{Z})$. If $\varphi=f_{*}$ for a homeomorphism $f$, then $\bar{\varphi}$ also preserves the (mod 2) intersection pairing on homology.

Our main result is that, for an automorphism $\varphi: H_{1}(F, \mathbb{Z}) \rightarrow H_{1}(F, \mathbb{Z})$, if the induced automorphism $\bar{\varphi}: H_{1}(F, \mathbb{Z} / 2 \mathbb{Z}) \rightarrow H_{1}(F, \mathbb{Z} / 2 \mathbb{Z})$ preserves the $(\bmod 2)$ intersection pairing, then $\varphi$ is induced by a homeomorphism of $F$.

Theorem 1.1. Let $\varphi: H_{1}(F, \mathbb{Z}) \rightarrow H_{1}(F, \mathbb{Z})$ be an automorphism. If the induced automorphism $\bar{\varphi}: H_{1}(F, \mathbb{Z} / 2 \mathbb{Z}) \rightarrow H_{1}(F, \mathbb{Z} / 2 \mathbb{Z})$ preserves the $(\bmod 2)$ intersection pairing, then $\varphi$ is induced by a homeomorphism of $F$.

We have a natural homomorphism $\operatorname{Aut}\left(H_{1}(F, \mathbb{Z})\right) \rightarrow \operatorname{Aut}\left(H_{1}(F, \mathbb{Z} / 2 \mathbb{Z})\right)$. Let $\mathscr{K}$ denote the kernel of this homomorphism, so that we have an exact sequence

$$
1 \rightarrow \mathscr{K} \rightarrow \operatorname{Aut}\left(H_{1}(F, \mathbb{Z})\right) \rightarrow \operatorname{Aut}\left(H_{1}(F, \mathbb{Z} / 2 \mathbb{Z})\right) \rightarrow 1 .
$$

Observe that elements of $\mathscr{K}$ automatically preserve the intersection pairing. We shall show that every element of $\mathscr{K}$ is induced by a homeomorphism of $F$. Further, we shall show that an element of $\operatorname{Aut}\left(H_{1}(F, \mathbb{Z} / 2 \mathbb{Z})\right)$ is induced by a homeomorphism of $F$ if and only if it preserves the intersection pairing. Theorem 1.1 follows immediately from these results.

Theorem 1.2. Suppose $\varphi: H_{1}(F, \mathbb{Z}) \rightarrow H_{1}(F, \mathbb{Z})$ is an automorphism which induces the identity on $H_{1}(F, \mathbb{Z} / 2 \mathbb{Z})$. Then $\varphi$ is induced by a homeomorphism of $F$. 
Theorem 1.3. Let $F_{1}$ and $F_{2}$ be closed, non-orientable surfaces. Suppose that $\psi: H_{1}\left(F_{1}, \mathbb{Z} / 2 \mathbb{Z}\right) \rightarrow H_{1}\left(F_{2}, \mathbb{Z} / 2 \mathbb{Z}\right)$ is an isomorphism which preserves the intersection pairing. Then $\psi$ is induced by a homeomorphism $f: F_{1} \rightarrow F_{2}$.

We also consider the case of a compact non-orientable surface $F$ with boundary. In this case an automorphism of $H_{1}(F, \mathbb{Z})$ induced by a homeomorphism of $F$ permutes (up to sign) the elements representing the boundary components. We shall show that all automorphisms of $H_{1}(F, \mathbb{Z})$ which satisfy this additional condition are induced by homeomorphisms. Other results regarding the homeomorphisms of non-orientable surfaces have been obtained by many authors, for instance [12]].

\section{Preliminaries}

Let $F$ be a closed, non-orientable surface with $\chi(F)=2-n$ and let $\hat{F}$ be obtained from $F$ by deleting the interior of a disc. Then $F$ is the connected sum of $n$ projective planes $\mathscr{P}_{i}$ and $\hat{F}$ is the boundary-connected sum of $n$ corresponding Möbius bands $\mathscr{M}_{i}$. Let $\gamma_{i}$ denote the central circle of $\mathscr{M}_{i}$ and let $\alpha_{i}=\left[\gamma_{i}\right] \in H_{1}(\hat{F}, \mathbb{Z})$ be the corresponding elements in homology. Then $H_{1}(\hat{F}, \mathbb{Z}) \cong \mathbb{Z}^{n}$ with basis $\alpha_{i}$ and $H_{1}(F, \mathbb{Z})$ is the quotient $H_{1}(\hat{F}, \mathbb{Z}) /\left\langle 2 \Sigma_{i} \alpha_{i}\right\rangle$.

We shall need the following elementary algebraic lemma.

Lemma 2.1. Any automorphism $\varphi: H_{1}(F, \mathbb{Z}) \rightarrow H_{1}(F, \mathbb{Z})$ lifts to an automorphism $\tilde{\varphi}: H_{1}(\hat{F}, \mathbb{Z}) \rightarrow H_{1}(\hat{F}, \mathbb{Z})$ such that $\tilde{\varphi}\left(\sum_{i} \alpha_{i}\right)=\sum_{i} \alpha_{i}$.

Proof. Consider the basis of $H_{1}(\hat{F}, \mathbb{Z})$ given by $e_{1}=\alpha_{1}, \ldots, e_{n-1}=\alpha_{n-1}, e_{n}=\alpha_{1}+\cdots+$ $\alpha_{n}$ and let $\left[e_{j}\right]$ be the corresponding generators of $H_{1}(F, \mathbb{Z})$. Observe that $\left[e_{n}\right]$ is the unique element of order 2 in $H_{1}(F, \mathbb{Z})$, and hence $\varphi\left(\left[e_{n}\right]\right)=\left[e_{n}\right]$. Thus, we can define $\tilde{\varphi}\left(e_{n}\right)=e_{n}$. For $1 \leq j \leq n-1$, pick an arbitrary lift $h_{j}$ of $\varphi\left(e_{j}\right)$ and set $\tilde{\varphi}\left(e_{j}\right)=h_{j}$.

Observe that $H_{1}(\hat{F}, \mathbb{Z}) /\left\langle e_{n}\right\rangle \cong H_{1}(F, \mathbb{Z}) /\left\langle\left[e_{n}\right]\right\rangle$. Further, as $\tilde{\varphi}\left(e_{n}\right)=e_{n}$ we have an induced map on $H_{1}(\hat{F}, \mathbb{Z}) /\left\langle e_{n}\right\rangle$ which agrees with the quotient map induced by $\varphi$ on $H_{1}(F, \mathbb{Z}) /\left\langle\left[e_{n}\right]\right\rangle$ (which exists as $\varphi\left(\left[e_{n}\right]\right)=\left[e_{n}\right]$ ) under the natural identification of these groups. As $\varphi$ is an isomorphism, so is the induced quotient map on $H_{1}(F, \mathbb{Z}) /\left\langle\left[e_{n}\right]\right\rangle$, and hence the map induced by $\tilde{\varphi}$ on $H_{1}(\hat{F}, \mathbb{Z}) /\left\langle e_{n}\right\rangle$.

Thus, $\tilde{\varphi}$ induces an isomorphism on the quotient $H_{1}(\hat{F}, \mathbb{Z}) /\left\langle e_{n}\right\rangle$ as well as the kernel $\left\langle e_{n}\right\rangle$ of the quotient map. By the five lemma, $\tilde{\varphi}$ is an isomorphism.

Henceforth, given an automorphism $\varphi$ as above, we shall assume that a lift has been chosen as in the lemma. Observe that a homeomorphism of $\hat{F}$ induces a homeomorphism of $F$. Hence it suffices to construct a homeomorphism of $\hat{F}$ inducing $\tilde{\varphi}$. Note that the intersection pairing is preserved by $\tilde{\varphi}$ as it only depends on the induced map on homology with $\mathbb{Z} / 2 \mathbb{Z}$-coefficients.

\section{Automorphisms in $\mathscr{K}$}

In this section we prove Theorem 1.2 Let $\varphi: H_{1}(F, \mathbb{Z}) \rightarrow H_{1}(F, \mathbb{Z})$ be as in the hypothesis. As in Lemma 2.1] we can lift $\varphi$ to an automorphism of $H_{1}(\hat{F}, \mathbb{Z})$ fixing $\sum_{i} \alpha_{i}$. We shall denote this lift also by $\varphi$. We shall construct a homeomorphism of $\hat{F}$ inducing this automorphism. 

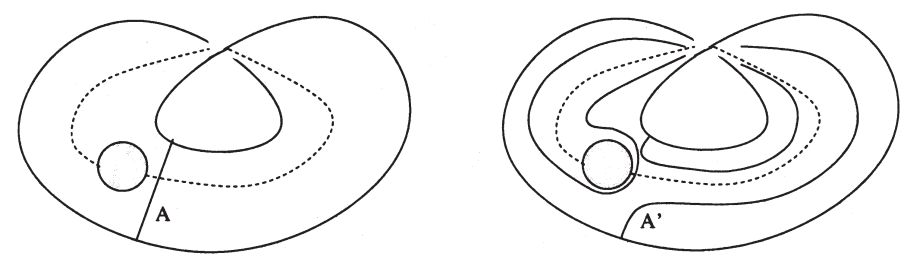

Figure 1. Cross-cap slide.

Our strategy is to use elementary automorphisms $e_{i j}, 1 \leq i, j \leq n$, which are induced by homeomorphisms $g_{i j}$. Observe that, for $1 \leq i, j \leq n$, the automorphism $\varphi$ is induced by a homeomorphism if and only if $e_{i j} \circ \varphi$ is induced by a homeomorphism (as $e_{i j}$ is induced by a homeomorphism). Thus we can replace $\varphi$ with $e_{i j} \circ \varphi$. We call this an elementary move. For $\varphi$ preserving the intersection pairing, we shall find a sequence of elementary moves such that on performing these moves we obtain the identity automorphism, which is obviously induced by a homeomorphism (namely the identity). This will prove the result.

Lemma 3.1. There are homeomorphisms $g_{i j}$ of $\hat{F}$ so that if $e_{i j}$ is the induced automorphism on $H_{1}(\hat{F}, \mathbb{Z})$, then $e_{i j}\left(\alpha_{i}\right)=\alpha_{i}+2 \alpha_{j}, e_{i j}\left(\alpha_{j}\right)=-\alpha_{j}$ and $e_{i j}\left(\alpha_{k}\right)=\alpha_{k}$ for $k \neq i, j$.

Proof. We shall use cross-cap slides [34] of the surface $F$. Namely, suppose $\alpha$ is an orientation reversing simple closed curve on a surface $S^{\prime}$ and $D$ is a small disc centered around a point on $\alpha$. Let $S$ be the surface obtained by replacing $D$ by a Möbius band. Consider a homeomorphism $f^{\prime}$ of $S^{\prime}$ which is the identity outside a neighbourhood of $\alpha$ and which is obtained by dragging $D$ once around $\alpha$ so that $D$ is mapped to itself. By construction this extends to a homeomorphism $f$ of $S$, which we call a cross-cap slide. In figure 1 the $\operatorname{arc} A$ in the Möbius band $\mathscr{M}$ on the left-hand side is mapped to the $\operatorname{arc} A^{\prime}$ in the Möbius band $\mathscr{M}^{\prime}$ on the right-hand side and the homeomorphism is the identity in a neighbourhood of the boundary.

We define $g_{i j}$ as the cross-cap slide of $\mathscr{M}_{j}$ around the curve $\gamma_{i}$. Note that the Möbius band $\mathscr{M}_{j}$ is mapped to itself, but, as $\gamma_{i}$ is orientation reversing, the map on the Möbius band takes $\alpha_{j}$ to $-\alpha_{j}$. Further for any $k$ different from $i$ and $j$, the cross-cap slide fixes $\gamma_{j}$, hence $\alpha_{j}$. Finally, in figure 1 (where we regard $\mathscr{M}$ as a neighbourhood of $\alpha_{j}$ ), if $B$ is a curve in the boundary of $\mathscr{M}$ joining the endpoints of $A$, then $[A \cup B]=\alpha_{i}$ and $\left[A^{\prime} \cup B\right]=g_{i j}\left(\alpha_{i}\right)$. It is easy to see that $[A \cup B]-\left[A^{\prime} \cup A\right]=\left[A \cup A^{\prime}\right]$ is homologous to the boundary $2 \alpha_{j}$ of the cross-cap. Thus, $e_{i j}\left(\alpha_{i}\right)=\alpha_{i}+2 \alpha_{j}$.

Lemma 3.2. There exists a sequence of elementary moves $e_{i j}$ taking $\varphi$ to the identity.

Proof. Let $\varphi$ be represented by a matrix $A=\left(a_{i j}\right)$ with respect to the basis $\alpha_{i}$. Then $A \equiv I(\bmod 2)$. As $\varphi$ fixes $\sum_{i} \alpha_{i}$, for every $i, \sum_{j} a_{i j}=1$. Observe that on performing the elementary move $e_{i j}$, the $i$ th column $A_{* i}$ of $A$ is replaced by $A_{* i}+2 A_{* j}$, the $j$ th column is replaced by $-A_{* j}$ and the other columns of $A$ are unchanged.

We first use the elementary moves $e_{i j}$ to reduce the first row $A_{1 *}$ to $[1,0,0, \ldots, 0]$. To do this, we define a complexity $C_{1}(A)$ of $A$ as $\left|a_{11}\right|+\left|a_{12}\right|+\cdots+\left|a_{1 n}\right|$.

Observe that if $a_{1 k}$ and $a_{1 l}$ are both non-zero, have different signs and $\left|a_{1 k}\right|>\left|a_{1 l}\right|$, $e_{k l}$ reduces the complexity $C_{1}(A)$. As $a_{11}$ is odd and $a_{1 j}$ is even for $j>1$, we know that 
$a_{11} \neq a_{1 j}$ for every $j>1$. Further, as $\sum_{j} a_{1 j}=1$, unless $a_{11}$ is 1 and $a_{1 j}=0$ for $j \neq 1$, there exists a $j>1$ such that $a_{11}$ and $a_{1 j}$ are of opposite signs (and both non-zero). Thus we can reduce complexity by performing an elementary operation. By iterating this finitely many times, we reduce the first row to $[1,0,0, \ldots, 0]$.

Next, suppose $i>1$ and the rows $A_{1 *}, A_{2 *}, \ldots, A_{(i-1) *}$ are the unit vectors $e_{1}, e_{2}, \ldots$, $e_{(i-1) *}$. We shall transform the $i$ th row to $[0,0, \ldots, 1,0, \ldots, 0]$ without changing the earlier rows.

First we shall transform the row $A_{i *}$ to a row of the form $[*, *, \ldots, 1,0, \ldots, 0]$ (i.e., with the first $i-1$ entries arbitrary) by performing elementary moves $e_{i j}$. To do this, we define a complexity $C_{i}(A)=\sum_{j}\left|a_{i j}\right|, j \geq i$.

Observe that, for $k \geq i$, the elementary operation $e_{1 k}$ changes the sign of $a_{i k}$, does not alter $a_{i m}$ for $m \neq k, m \geq i$ and does not change first $i-1$ rows. By such operations we can ensure that $a_{i i}>0$ and $a_{i j} \leq 0$ for $j>i$ without changing the complexity.

As before, $a_{i i} \neq a_{i j}$ for $j>i$ (as $a_{i i}$ is odd and $a_{i j}$ is even) and (using operations $e_{1 k}$ if necessary) $a_{i i}$ and $a_{i j}$ have different signs. Hence, unless $a_{i j}=0$ for $j>i$ we can reduce the complexity using either $e_{i j}$ or $e_{j i}$, without altering the first $i$ rows. Thus we can reduce $A_{i *}$ to a vector of the form $[*, \ldots, *, m, 0, \ldots, 0]$.

Now $A$ is a block lower triangular matrix with $a_{i i}$ as a diagonal entry. As $A$ is invertible it follows that $m=a_{i i}= \pm 1$.

We define another complexity $C_{i}^{\prime}(A)=\sum_{i \geq j}\left|a_{i j}\right|$. As $\sum_{j} a_{i j}=1$ and $a_{i i}= \pm 1$, unless $A_{i *}$ is a unit vector we can find as before an operation $e_{j i}, j<i$, which reduces this complexity (without changing the first $(i-1)$ rows). Hence after finitely many steps the $i$ th row is reduced to a unit vector. By applying these moves for $i=2,3, \ldots, n$, we are done.

\section{Automorphisms of $H_{1}(F, \mathbb{Z} / 2 \mathbb{Z})$}

We now prove Theorem 1.3 We shall proceed by induction on $n$. In the case when $n=1$ the result is obvious. We henceforth assume that $n$ is greater than 1 .

We first make some observations. For a surface $S$, any element $\alpha$ of $H_{1}(S, \mathbb{Z} / 2 \mathbb{Z})$ can be represented by a simple closed curve. The curve $\alpha$ is orientation reversing if and only if $\alpha \cdot \alpha=1$. The surface is non-orientable if and only if there exist $\alpha \in H_{1}(S, \mathbb{Z} / 2 \mathbb{Z})$ with $\alpha \cdot \alpha=1$.

As before, let $F_{1}$ be the connected sum of $\mathscr{P}_{1}, \mathscr{P}_{2}, \ldots, \mathscr{P}_{n}$, where $\mathscr{P}_{i}$ denotes a projective plane and $\mathscr{M}_{i}$ denotes the corresponding Möbius band. Let $\alpha_{1}, \alpha_{2}, \ldots, \alpha_{n}$ and $\gamma_{1}, \ldots, \gamma_{n}$ be as before.

Let $\psi$ be as in the hypothesis. Let $\beta_{i}=\psi\left(\alpha_{i}\right)$ and let $C$ be a simple close curve that represents $\beta_{1}$. As $\beta_{1} \cdot \beta_{1}=\alpha_{1} \cdot \alpha_{1}=1, C$ is orientation reversing (as is $\gamma_{1}$ ). Hence regular neighbourhoods of $C$ and $\gamma_{1}$ are Möbius bands.

Let $\hat{F}_{1}^{\prime}=F_{1}-\operatorname{int}\left(\mathscr{N}\left(\gamma_{1}\right)\right)$ and $\hat{F}_{2}^{\prime}=F_{2}-\operatorname{int}(\mathscr{N}(C))$. Let $F_{1}^{\prime}=\hat{F}_{1}^{\prime} \cup D^{2}$ and $F_{2}^{\prime}=\hat{F}_{2}^{\prime} \cup D^{2}$ be closed surfaces obtained by capping off $\hat{F}_{i}$.

Observe that the surface $F_{1}^{\prime}$ is non-orientable as $n \geq 2$ and $\gamma_{2}$ is an orientation reversing curve on it. Now since $\psi$ preserves the intersection pairing it takes orthonormal basis of $H_{1}\left(F_{1}, \mathbb{Z} / 2 \mathbb{Z}\right)$ to orthonormal basis of $H_{1}\left(F_{2}, \mathbb{Z} / 2 \mathbb{Z}\right)$. It follows that $\beta_{j} \cdot \beta_{j}=1$ for every $j$. Further, by a Mayer-Vietoris argument, $H_{1}\left(F_{i}, \mathbb{Z} / 2 \mathbb{Z}\right)=\mathbb{Z} / 2 \mathbb{Z} \oplus H_{1}\left(F_{i}^{\prime}, \mathbb{Z} / 2 \mathbb{Z}\right)$, with the decomposition being orthogonal and the component $\mathbb{Z} / 2 \mathbb{Z}$ in $H_{1}\left(F_{1}, \mathbb{Z} / 2 \mathbb{Z}\right.$ ) (respectively $H_{1}\left(F_{2}, \mathbb{Z} / 2 \mathbb{Z}\right)$ ) is spanned by $\alpha_{1}$ (respectively $\beta_{1}$ ). As $\psi$ preserves the intersection pairing, it follows that $\psi$ induces an isomorphism $\psi: H_{1}\left(F_{1}, \mathbb{Z} / 2 \mathbb{Z}\right) \rightarrow H_{1}\left(F_{2}, \mathbb{Z} / 2 \mathbb{Z}\right)$. 
Hence if $C_{2}$ is a curve in $F_{2}^{\prime}$ representing $\beta_{2}$ in $H_{1}\left(F_{2}, \mathbb{Z} / 2 \mathbb{Z}\right)$, then $C_{2}$ is orientation reversing and hence $F_{2}^{\prime}$ is non-orientable. Also, we have seen that the map $\psi$ induces an isomorphism from $H_{1}\left(F_{1}^{\prime}, \mathbb{Z} / 2 \mathbb{Z}\right)$ to $H_{1}\left(F_{2}^{\prime}, \mathbb{Z} / 2 \mathbb{Z}\right)$. By the induction hypothesis such a map is induced by a homoeomorphism $f^{\prime}: F_{1}^{\prime} \rightarrow F_{2}^{\prime}$.

Note that $F_{1}$ (respectively $F_{2}$ ) is obtained from $F_{1}^{\prime}$ (respectively $F_{2}^{\prime}$ ) by deleting the interior of a disc $D_{1}$ (respectively $D_{2}$ ) and gluing in $\mathscr{N}\left(\gamma_{1}\right)$ (respectively $\mathscr{N}(C)$ ). We can modify $f^{\prime}$ so that $f^{\prime}\left(D_{1}\right)=D_{2}$. On $F_{1}-\operatorname{int}\left(D_{1}\right)$ we define $f=f^{\prime}$. This restricts to a homeomorphism mapping $\partial \mathscr{N}\left(\gamma_{1}\right)$ to $\partial \mathscr{N}(C)$, which extends to a homeomorphism mapping $\mathscr{N}\left(\gamma_{1}\right)$ to $\mathscr{N}(C)$. As $\left.f\right|_{\mathscr{N}\left(\gamma_{1}\right)}: \mathscr{N}\left(\gamma_{1}\right) \rightarrow \mathscr{N}(C)$ is a homeomorphism, it maps the generator $\alpha_{1}$ of $H_{1}\left(\mathscr{N}\left(\gamma_{1}\right), \mathbb{Z}\right)=\mathbb{Z}$ to a generator $\pm \beta$ of $H_{1}(\mathscr{N}(C), \mathbb{Z})=\mathbb{Z}$. Thus with $\bmod 2$ coefficients, $f_{*}=\varphi$ as required.

\section{An algebraic corollary}

We shall deduce from Theorem 1.1 and a theorem of Lickorish [3] a purely algebraic corollary. While this has a straightforward algebraic proof (and is presumably wellknown), it may still be of interest to see its relation to topology.

Let $V=(\mathbb{Z} / 2 \mathbb{Z})^{n}$ be a vector space over $\mathbb{Z} / 2 \mathbb{Z}$ and let $\left\{e_{j}\right\}$ be the standard basis of $V$. Consider the standard inner product $\left\langle\left(x_{i}\right),\left(y_{i}\right)\right\rangle=\sum_{i} x_{i} y_{i}$. Let $\mathscr{O}$ be the group of automorphisms of $V$ that preserve the inner product. We shall show that $\mathscr{O}$ is generated by certain involutions.

Namely, let $1 \leq i_{1}<i_{2}<\cdots<i_{2 k} \leq n$ be $2 k$ integers between 1 and $n$. We define an element $R=R\left(i_{1}, \ldots, i_{2 k}\right)$ to be the transformation defined by

$$
\begin{aligned}
& R\left(e_{i_{j}}\right)=\sum_{l \neq j} e_{i_{l}}, \\
& R\left(e_{j}\right)=e_{j}, j \neq i_{1}, i_{2}, \ldots i_{2 k} .
\end{aligned}
$$

Theorem 5.1. The group $\mathscr{O}$ is generated by the involutions $R\left(i_{1}, \ldots, i_{2 k}\right)$.

Proof. We identify $V$ with $H_{1}(F, \mathbb{Z} / 2 \mathbb{Z})$ for a non-orientable surface $F$ and identify the basis elements $e_{i}$ with $\alpha_{i}$. Under this identification, the bilinear pairing on $V$ corresponds to the intersection pairing. We shall see that the transformations $R\left(i_{1}, \ldots, i_{2 k}\right)$ correspond to the action of Dehn twists on $H_{1}(F, \mathbb{Z} / 2 \mathbb{Z})$, where we identify the generators $e_{i}$ with $\alpha_{i}$. First note that any element $\gamma$ of $H_{1}(F, \mathbb{Z} / 2 \mathbb{Z})$ can be expressed as $\gamma=\alpha_{i_{1}}+\cdots+\alpha_{i_{m}}$. Observe that a simple closed curve $C$ representing $\gamma$ is orientation preserving if and only if $\gamma \cdot \gamma=0$, which is equivalent to $m$ being even.

Now let $C$ be an orientation preserving curve on $F$ and consider the Dehn twist $\tau$ about $C$. Let $\gamma=[C] \in H_{1}(F, \mathbb{Z} / 2 \mathbb{Z})$ be the element represented by $C$. By the above (as $\gamma=\alpha_{i_{1}}+\cdots+\alpha_{i_{m}}$ and $m$ is even), we can express $\gamma$ as $\gamma=\alpha_{i_{1}}+\cdots+\alpha_{i_{2 k}}$. If $\alpha$ is another element of $H_{1}(F, \mathbb{Z} / 2 \mathbb{Z})$ and $\alpha \cdot \gamma$ is the $(\bmod 2)$ intersection number, then (with $\bmod 2$ coefficients) $\tau_{*}(\alpha)=\alpha+\gamma$. It is easy to see that $\tau_{*}=R\left(i_{1}, \ldots, i_{2 k}\right)$. Note that $\tau_{*}^{2}(\alpha)=$ $\alpha+2 \gamma=\alpha$, hence $\tau_{*}=R\left(i_{1}, \ldots, i_{2 k}\right)$ is an involution as claimed.

Now, by Theorem 1.3 any element $\phi \in \mathscr{O}$ is induced by a homeomorphism $f$ of $F$. Further, by a theorem of Lickorish [3], $f$ is homotopic to a composition of Dehn twists and cross-cap slides. We have seen that Dehn twists induce the automorphisms $\tau_{*}=R\left(i_{1}, \ldots, i_{2 k}\right)$ on $V$. It is easy to see that cross-cap slides induce the identity on $H_{1}(F, \mathbb{Z} / 2 \mathbb{Z})$. Thus $\phi$ is a composition of elements of the form $\tau_{*}=R\left(i_{1}, \ldots, i_{2 k}\right)$ as claimed. 
Remark 5.2. We can alternatively deduce Theorem 1.3 from Theorem 5.1 as the generators of $\mathscr{O}$ can be represented by homeomorphisms (namely Dehn twists).

\section{Punctured surfaces}

Let $F$ be a compact non-orientable surface with $m$ boundary components and let $\beta_{j} \in$ $H_{1}(F, \mathbb{Z}), 1 \leq j \leq m$, be elements representing the boundary curves. A homeomorphism $f: F \rightarrow F$ induces an automorphism $\varphi=f_{*}$ of $H_{1}(F, \mathbb{Z})$. Furthermore, as boundary components of $F$ are mapped to boundary components by $f$ (possibly reversing orientations), for some permutation $\sigma$ of $\{1, \ldots, m\}$ and some constants $\varepsilon_{j}= \pm 1, \varphi\left(\beta_{j}\right)=\varepsilon_{j} \beta_{\sigma(j)}$, for all $j, 1 \leq j \leq m$.

We show that conversely any automorphism $\varphi$ that preserves the (mod 2) intersection pairing and takes boundary components to boundary components is induced by a homeomorphism.

Theorem 6.1. Let $F$ be a compact non-orientable surface with $m$ boundary components and let $\varphi$ be an automorphism of $H_{1}(F, \mathbb{Z} / 2 \mathbb{Z})$ that preserves the (mod 2) intersection pairing. Suppose for some permutation $\sigma$ of $\{1, \ldots, m\}$ and some constants $\varepsilon_{j}= \pm 1$, we have $\varphi\left(\beta_{j}\right)=\varepsilon_{j} \beta_{\sigma(j)}$, for all $1 \leq j \leq m$. Then $\varphi$ is induced by a homeomorphism of $F$.

Proof. Let $\bar{F}$ be obtained from $F$ by attaching discs to all the boundary components. Then we can assume that $F$ has been obtained from $\bar{F}$ by deleting the interiors of $m$ discs $D_{1}, \ldots D_{m}$, all of which are contained in a disc $E \subset \bar{F}$. Further we can assume that the central curves $\gamma_{i}, 1 \leq i \leq n$ in a decomposition of $\bar{F}$ into projective planes are disjoint from $E$, as are all the Dehn twists and cross-cap slides we perform on $\bar{F}$ in the proof of Theorem 1.1 Hence the Dehn twists and cross-cap slides we perform give homeomorphisms of $F$ which are the identity on the boundary components.

Let $\alpha_{i}=\left[\gamma_{i}\right]$ and let $\bar{\alpha}_{i}$ be the images of these elements in $H_{1}(\bar{F}, \mathbb{Z})$. By choosing appropriate orientations, we get that $H_{1}(F, \mathbb{Z})$ is generated by the elements $\alpha_{i}$ and $\beta_{j}$ with the relation

$$
2 \sum_{i} \alpha_{i}=\sum_{j} \beta_{j}
$$

Note that as $H_{1}(\bar{F}, \mathbb{Z})=H_{1}(F, \mathbb{Z}) /\left\langle\beta_{j}\right\rangle$, it follows by the hypothesis that $\varphi$ induces an automorpism $\bar{\varphi}$ of $H_{1}(\bar{F}, \mathbb{Z})$. By Theorem 1.1 (and its proof), this is induced by a composition of Dehn twists and cross-cap slides, hence a homeomorphism $g: F \rightarrow F$. By composing $\varphi$ by $g_{*}^{-1}$, we can assume that $\bar{\varphi}$ is the identity.

Similarly, we can use homeomorphisms supported in $E$ (which do not change any $\alpha_{i}$ ) to reduce to the case when the permutation $\sigma$ is the identity, i.e. $\varphi\left(\beta_{j}\right)=\varepsilon_{j} \beta_{j}$. As $\bar{\varphi}\left(\bar{\alpha}_{j}\right)=$ $\bar{\alpha}_{j}$, we get $\varphi\left(\alpha_{i}\right)=\alpha_{i}+\sum_{j} c_{i j} \beta_{j}$ for some integers $c_{i j}$. We define the complexity of $\varphi$ to be $C(\varphi)=\sum_{i, j}\left|c_{i j}\right|$.

If $\varphi$ is not the identity, we shall reduce the complexity of $\varphi$ using homeomorphisms called boundary slides [2] similar to cross-cap slides.

Lemma 6.2. There are homeomorphisms $h_{i j}$ of $F$ such that the induced automorphism of $H_{1}(F, \mathbb{Z})$ takes $\alpha_{i}$ to $\alpha_{i}-\beta_{j}$, maps $\beta_{j}$ to $-\beta_{j}$ and fixes all other $\alpha$ 's and $\beta$ 's.

Proof. We shall use boundary slides [2] of the surface $F$. Namely, suppose $\alpha$ is an orientation reversing simple closed curve on a surface $S^{\prime}$ and $D$ is a small disc centered around 
a point on $\alpha$. Let $S$ be the surface obtained by deleting the interior of $D$. Consider a homeomorphism of $S^{\prime}$ which is the identity outside a neighbourhood of $\alpha$ and which is obtained by dragging $D$ once around $\alpha$ so that $D$ is mapped to itself. By construction this extends to a homeomorphism of $S$, which we call a boundary slide.

As in the case of cross-cap slides, the automorphism of $H_{1}(F, \mathbb{Z})$ induced by the boundary slide of the boundary component corresponding to $\beta_{j}$ along the simple closed curve $\gamma_{i}$ (representing $\alpha_{i}$ ) is as in the statement of the lemma.

Now suppose $\varphi$ is not the identity. Observe that as $\varphi$ is a homomorphism, $2 \sum_{i} \varphi\left(\alpha_{i}\right)=$ $\sum_{j} \varphi\left(\beta_{j}\right)$. Using $\varphi\left(\alpha_{i}\right)=\alpha_{i}+\sum_{j} c_{i j} \beta_{j}, \varphi\left(\beta_{j}\right)=\varepsilon_{j} \beta_{j}$ and $2 \sum_{i} \alpha_{i}=\sum_{j} \beta_{j}$, we see that $\sum_{j} c_{i j} \beta_{j}=\left(\varepsilon_{j}-1\right) \beta_{j}$. As the elements $\beta_{j}, 1 \leq j \leq n$ are independent, it follows that for each $j, \Sigma_{i} c_{i j}=\varepsilon_{j}-1$.

We now consider two cases. Firstly, if some $\varepsilon_{j}=-1$, then observe that postcomposing with $h_{i j}$ takes $\varphi\left(\alpha_{i}\right)$ to $\varphi\left(\alpha_{i}\right)-\varphi\left(\beta_{j}\right)=\varphi\left(\alpha_{i}\right)+\beta_{j}$. Hence $c_{i j}$ is changed to $c_{i j}+1$ (and no other $c_{k l}$ is changed). In particular, if $c_{i j}<0$, the complexity is reduced. But as $\sum_{i} c_{i j}=$ $\varepsilon_{j}-1=-2$, we must have some $c_{i j}<0$, and hence a move reducing complexity.

Suppose now that each $\beta_{j}$ is 1 . Then as $\sum_{i} c_{i j}=\varepsilon_{j}-1=0$, either each $c_{i j}=0$, in which case we are done, or some $c_{i j}>0$. Observe that postcomposing with $h_{i j}$ takes $\varphi\left(\alpha_{i}\right)$ to $\varphi\left(\alpha_{i}\right)-\varphi\left(\beta_{j}\right)=\varphi\left(\alpha_{i}\right)-\beta_{j}$. Hence $c_{i j}$ is changed to $c_{i j}-1$ (and no other $c_{k l}$ is changed), and hence the complexity is reduced. Thus in finitely many steps, we reduce to the case where $\varphi$ is the identity.

\section{Acknowledgements}

We would like to thank Shreedhar Inamdar for helpful conversations.

\section{References}

[1] Birman J S and Chillingworth D R J, On the homotopy group of a non-orientable surface, Math. Proc. Cambridge Philos. Soc. 136 (1972) 437-448

[2] Korkmaz M, Mapping class groups of nonorientable surfaces, Geometriae Dedicata 89 (2002) 109-133

[3] Lickorish W B R, Homeomorphisms of non-orientable two-manifold, Math. Proc. Cambridge Philos. Soc. 59 (1963) 307-317

[4] Lickorish W B R, Homeomorphisms of non-orientable two-manifold, Math. Proc. Cambridge Philos. Soc. 61 (1965) 61-64 